\title{
The Importance of Multidisciplinary Approach in Intensive Care Unit: Conservative Management of Two Cases Following Isolated Splenic Rupture Due to Blunt Abdominal Injury
}

\author{
Öznur ULUDAĞ ${ }^{1}$, Sadık ÇAĞ² ${ }^{2}$ Ali Haydar BAYKAN³ , Ülkü SABUNCU¹, Hatice KUŞDERCi ${ }^{1}$
}

\footnotetext{
${ }^{1}$ Department of Anesthesiology and Reanimation, Adiyaman University Research and Educational Hospital,Adiyaman, Turkey.

${ }^{2}$ Department of General Surgery Adiyaman University Research and Educational Hospital,Adiyaman, Turkey.

${ }^{3}$ Department of Radiological Adiyaman University Research and Educational Hospital,Adiyaman, Turkey.
}

\section{ABSTRACT}

In the recent years, treatment strategy for hemodynamically stable patients with splenic trauma has changed to nonoperative therapy as a result of the increased quality of intensive care units (ICUS) and advances in imaging techniques for the diagnosis of the solid-organ traumas. This case report presented the conservative approach for two patients who had isolated splenic trauma in the ICU. The report was aimed to emphasize the importance of multidisciplinary approach in ICUs to increase the quality of service provided.

Key words: Conservative treatment, intensive care, multidisciplinary approach, splenic rupture

\section{INTRODUCTION}

Different approaches have been used for the treatment of solid-organ injuries after blunt abdominal traumas. Surgical interventions such as primary repair of the injured organ, whole or partial removal of the organ, electrocauterization, and hemostasis have been applied as standard treatments for years. Diagnostic and follow-up methods have become faster and less invasive with advancing technology and growing body of knowledge.

Structuring and managing intensive care units (ICUs) under the supervision of an Anesthesiology and Reanimation specialist or an Intensive Care specialist enables staff education, development of clinical protocols for critical patient care, and reliable and high-quality patient care in these units.

This report presented two cases of spleen injury treated conservatively without surgery by cooperation of an anesthesiologist and a general surgeon at an ICU.

\section{CASE 1}

A 24-year-old male patient who met with a traffic accident was evaluated at the emergency department. His physical examination revealed that his general condition was good, he was conscious, pupils were normoisocoric, Glasgow Coma Scale score was 15, and respiration was normal. His medical history was unremarkable. Arterial blood pressure was 120/70 $\mathrm{mmHg}$, pulse rate was 80 beats/minute, and pulses were rhythmic. Posterioanterior (PA) lung $x$-ray was unremarkable. Sensitivity was detected at abdominal examination. Hematocrit value was 45. In his computed tomography scannig (CT) especially at inferior anterior face, any enhanced contrast was observed. This area was observad as devascularized, geografic shaped and had hipodens; also splenic vein and hilus was preserved (Figure 1). It was interpreted as grade 4 spleen injury. The patient was hemodynamically stable. So, conservative treatment was planned and he was admitted to the ICU. For first $24 \mathrm{~h}$, arterial tension, pulse rate, peripheral oxygen saturation, and urine output follow-up were performed every hour, and hematocrit and leukocyte follow-up and abdominal examination were performed every 6 
hours. The patient stayed at the ICU for 3 days. On the third day, oral intake was permitted. He was followed up in the surgery ward for 5 days and discharged. Abdominal ultrasonography 6 months after discharge revealed that spleen contours were regular and echogenicity of the parenchyma was homogenous (Figure 2). Traumatic areas described by CT disappeared. He was considered as totally cured.

\section{CASE 2}

A 57-year-old male who met with an in-vehicle traffic accident was evaluated at the emergency department. His general condition was good, sensation was open, and orientation and cooperation were intact. Arterial blood pressure was 110/70 $\mathrm{mmHg}$, and pulse rate was 85 beats/minute. Abdominal tenderness was present. In his computed tomography scanning at posterior region of spleen, nearly $1 \mathrm{~cm}$ pyramid shaped laceration that was penatrated to spleen paranchyme was observed (Figure 3). Free liquid was observed in perihepatic and perisplenic spaces. Liquid densities representing contusion were present on the posterior sides of both lungs, and atelectatic areas were present in the neighboring areas. Conservative approach was planned, and the patient was admitted to the ICU. He was treated for 2 days at the ICU. Then, he was followed up in the surgery ward and discharged.

Classification was made according to the Abbreviated Injury Scale (AIS) for splenic injuries (Table 1).

\section{DISCUSSION}

Splenectomy for spleen injury has been used as a safe method for years. However, more recently, understanding of the importance of spleen in human immune system, increased utilization of hemostatic agents, demonstration of the safety of spleensparing methods such as splenorrhaphy and partial splenectomy, and advances in patient care have limited the use of splenectomy for traumatic spleen injuries (1). Previously, a positive result in a diagnostic peritoneal lavage was considered to be an important marker of intra-abdominal hemorrhage, and accepted as an indication for laparotomy. However, successful results achieved by nonoperative treatment of splenic injuries in children led to the idea of using the same approach in adolescents (2).
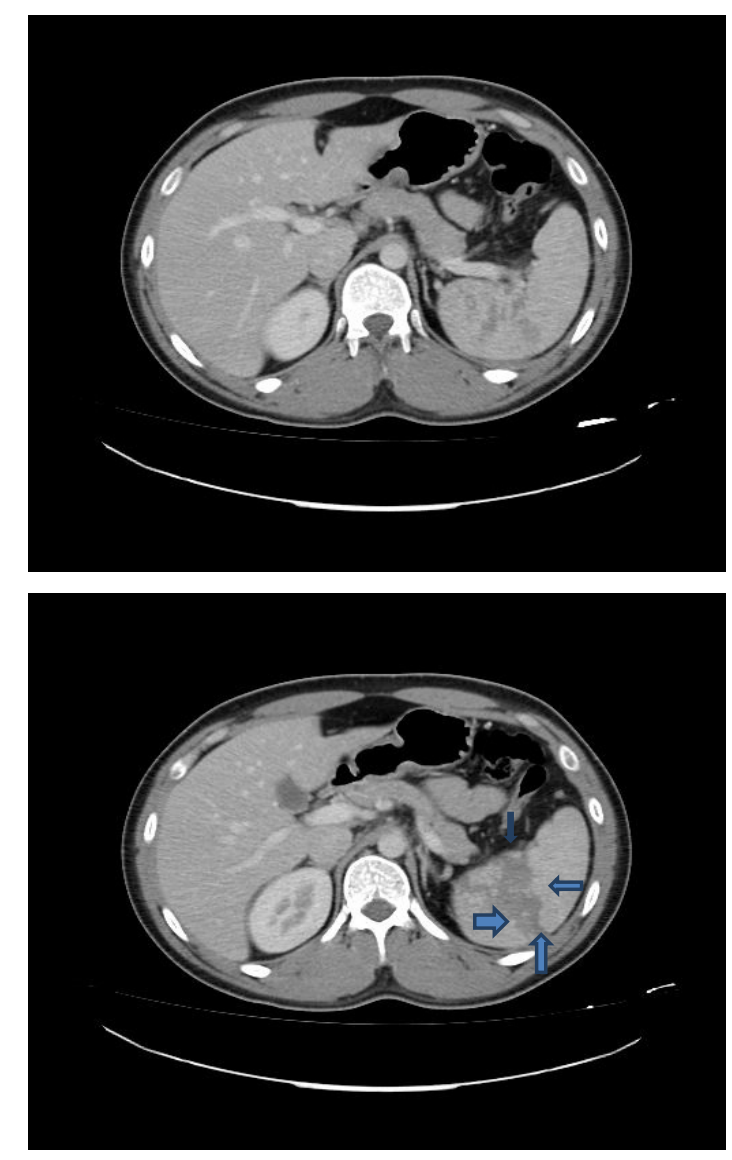

FigurE 1: $(A, B)$ Cross-section from the first CT imaging of the patient (Case 1).

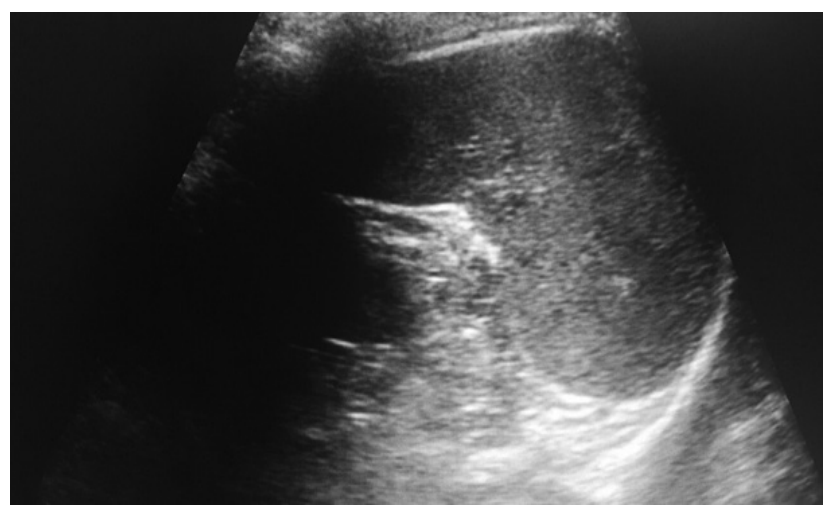

FIGURE 2: Control ultrasound evaluation 6 months after trauma.

Conservative treatment approach for spleen injuries is important to prevent risks and possible complications due to anesthesia, risk of incisional herniation and intra-abdominal adhesions after surgery, high cost due to operation, increased hospitalization and hence absenteeism from work leading to economic losses, increased risk of infections in splenectomized patients and 


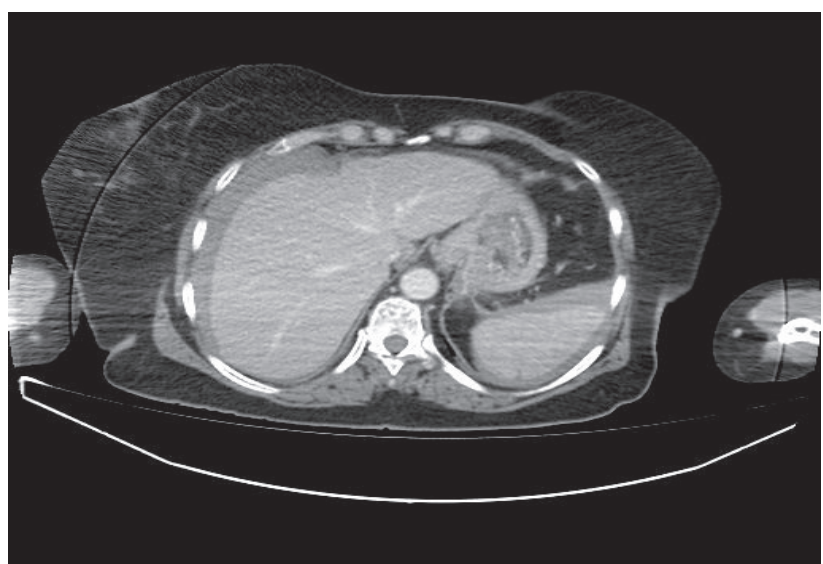

FIGURE 3: Cross -section showing first CT evaluation of the patient (Case 2).

severity of sepsis that can develop after splenectomy, and complications that may develop after splenectomy. Compared with surgical treatment, lower complication rate and lower cost are some of the advantages of conservative treatment (3).

Hemodynamically stable patients may be treated without splenectomy after careful clinical and radiological evaluation. The most debated topic about imaging in conservative management is determination of the roles of $\mathrm{CT}$ and ultrasound in grading and follow-up of solid-organ injuries. Ultrasound has been used increasingly for the initial evaluation and follow-up of abdominal traumas because it is noninvasive, does not involve radiation, is cheap, can be performed in a short time, and can be easily applied. If intra-abdominal free-fluid or solid-organ injuries are detected, $\mathrm{CT}$ is performed in hemodynamically stable patients to be sure about the presence of injury and to grade the injury (4). In these cases, CT was used for initial evaluation and grading and ultrasound was used at 6-month follow-up due to its advantages.

Although conservative follow-up is a method associated with low mortality and morbidity, it should not be kept in mind that clinical findings warranting surgical intervention may develop during follow-up. The chief physician of the ICU has an important responsibility to follow these findings. At this point it can be said that, multidisciplinary approach at patient followup is so importance. Additionally, multidisciplinary follow-up enables multiple disciplines to fulfill their responsibilities timely. Abundant evidence supports the involvement of clinicians experienced in intensive unit care, especially for critically ill patients. Many studies have shown that an experienced clinician in the ICU increases the success rate and decreases the cost. A large meta-analysis evaluated the association between the involvement of experienced clinicians in the ICU and clinical courses of critical cases (5). A comparison of low-intensity ICUs in which consultation to ICU specialist is not made or electively made, with high-intensity ICUs in which consultation to ICU specialist is mandatory or ICUs working in a "closed" model demonstrated that hospital mortality was 29\% lower and ICU mortality was 39\% lower in high-intensity ICUs. Carson et al. (6) prospectively evaluated the effects of transition from an "open" ICU model in which clinicians from various disciplines care for patients from their own specialty without support from ICU

TABLE 1: Abbreviated Injury Scale (AIS) system for the classification of splenic injuries

\begin{tabular}{ll}
\hline GRADE 1 & Hematoma subcapsular, does not increase, smaller than $10 \%$ of the surface area. \\
& Laceration capsular tears, not blood, parenchymal depth of less than $1 \mathrm{~cm}$. \\
\hline GRADE 2 & Hematoma subcapsular, does not increase, until $10-50 \%$ of the surface area. \\
& Laceration capsular tear, active bleeding, parenchymal depth of $1-3 \mathrm{~cm}$, length $<10 \mathrm{~cm}$. \\
\hline GRADE 3 & Hematoma subcapsular, larger than $50 \%$ of the surface area or increasing. \\
& $\begin{array}{l}\text { Active bleeding subcapsular hematoma rupture. } \\
\text { Large or growing intraparenchymal hematoma larger than } 2 \mathrm{~cm} .\end{array}$ \\
& Parenchymal laceration depth of more than $3 \mathrm{~cm}$. \\
\hline GRADE 4 & Hematoma subcapsular. Active bleeding rupture. \\
& Laceration bleeding vessels in the segment or hilar devascularization $>25 \%$. \\
\hline GRADE 5 & Laceration complex multipart. \\
& Hilar vessels full devascularization, vascular injury.
\end{tabular}


specialists, to a "closed" model in which patient care is under the supervision of ICU specialists. Without using additional resources, significant decreases were observed in scores determining the mortality risk. In a retrospective study, Ghorra et al. (7) demonstrated a decrease in mortality and complications after changing open ICUs to closed ones.

In the aforementioned cases, grade 4 and grade 1 spleen injuries were nonoperatively managed and the patients were discharged with cure using a multidisciplinary approach under the supervision of an anesthesiologist. Successful application of up-to-date diagnostic and treatment approaches in the ICU reflects the importance of team work.

\section{CONCLUSION}

It is believed that health policies should be implemented that enable a multidisciplinary team approach under the leadership of experienced ICU clinicians, especially anesthesiologists, in order to improve the health care quality in ICUs.

\section{REFERENCES}

1. Morrell DG, Chang FC, Helmer SD. Changing trends in the management of splenic injury. Am JSurg 1995; 170:686-689.

2. Ertekin C. Solid organ yaralanmalarında konservatif tedavi ilkeleri. Türkiye Klinikleri J Surg Med Sci 2004;9:212-217.

3. Livingston DH, Tortella BJ, Blackwood J, Marchiedo GW, Rush BF Jr. The role of laparoscopy in abdominal trauma. J Trauma 1992;33:471-475.

4. Rutledge $R$, Hunt JP, Lentz CW, Fakhry SM, Meyer AA, Baker $\mathrm{CC}$ et al. A statewide, population-based time series analysis of the increasing frequency of nonoperative management of abdominal solid organ injury. Ann Surg 1995; 222:311-316.

5. Pronovost PJ, Angus DC, Dorman T, et al. Physiian staffing patterns and clinical outcomes in critically ill patients: A systematic rewiew. JAMA 2002;288:2151-2162.

6. Carson SS, Stocking C, Podsadecki T, et al. Effects of organizational change in the medical intensive care unit of a teaching hospital. JAMA 1996; 276:322-328.

7. Ghorra S, Reinert SE, Cioffi W, et al. Analysis of the effect of conversion from open to closed surgical intensive care unit. Ann Surg 1999; 229:163-171. 